\title{
Evaluation of correlative coding and DP-16QAM n-channel 112Gbit/s coherent transmission: digital non-linear compensation perspective
}

\author{
Rameez Asif,,${ }^{1,2,3, *}$ Chien-Yu Lin, ${ }^{1,2}$ Michael Holtmannspoetter, ${ }^{1,2}$ and \\ Bernhard Schmauss ${ }^{1,2}$ \\ ${ }^{1}$ Institute of Microwaves and Photonics, University of Erlangen (FAU), \\ Erlangen (91058), Germany \\ ${ }^{2}$ Erlangen Graduate School in Advanced Optical Technologies (SAOT), \\ Erlangen (91052), Germany \\ ${ }^{3}$ Telecommunication Engineering Department (TED), University of Engineering and \\ Technology (UET), Taxila (47050), Pakistan \\ *rameez@lhft.eei.uni-erlangen.de,rameez.asif@uettaxila.edu.pk
}

\begin{abstract}
We numerically report on the complexity reduction of digital backward propagation (DBP) by utilizing correlative encoded transmission (dual-polarization quadrature duobinary) at a bit-rate of $112 \mathrm{Gbit} / \mathrm{s}$ over $1640 \mathrm{~km}$ fibe link. The single channel $(\mathrm{N}=1)$ and multi-channel $(\mathrm{N}=10)$ transmission performances are compared in this paper. In case of multichannel system, 10 transmitters are multiplexed with $25 \mathrm{GHz}$ channel spacing. The fibe link consists of Large $\mathrm{A}_{e f f}$ Pure-Silica core fibe with 20 spans of $82 \mathrm{~km}$ each. No in-line optical dispersion compensator is employed in the link. The system performances are evaluated by monitoring the bit-error-ratio and the forward error correction limit corresponds to bit-error-ratio of $3.8 \times 10^{-3}$. The DBP algorithm is implemented after the coherent detection and is based on the logarithmic step-size based splitstep Fourier method. The results depict that dual-polarization quadrature duobinary can be used to transmit $112 \mathrm{Gbit} / \mathrm{s}$ signals with an spectral efficien $\mathrm{y}$ of $4-\mathrm{b} / \mathrm{s} / \mathrm{Hz}$, but at the same time has a higher tolerance to nonlinear transmission impairments. By utilizing dual-polarization quadrature duobinary modulation, comparative system performance with respect to dual-polarization 16-quadrature amplitude modulation transmission can be achieved with $60 \%$ less computations and with a step-size of $205 \mathrm{~km}$.
\end{abstract}

(C) 2013 Optical Society of America

OCIS codes: (060.2330) Fiber optics communications; (060.1660) Coherent communications; (190.4370) Nonlinear optics, fibers

\section{References and links}

1. X. Li, X. Chen, G. Goldfarb, E. Mateo, I. Kim, F. Yaman, and G. Li, "Electronic post-compensation of WDM transmission impairments using coherent detection and digital signal processing," Opt. Express 16, 880-888 (2008).

2. E. Ip, and J.M. Kahn, "Compensation of dispersion and non-linear impairments using digital backpropagation," J. Lightwave Technol. 26(20), 3416-3425 (2008).

3. D. S. Millar, S. Makovejs, C. Behrens, S. Hellerbrand, R. Killey, P. Bayvel, and S. Savory, "Mitigation of fibe non-linearity using a digital coherent receiver," IEEE J. Sel. Top. Quantum Electron. 16(5), 1217-1226 (2010). 
4. C. R. S Fludger, T. Duthel, D. van den Borne, C. Schulien, E. Schmidt, T. Wuth, J. Geyer, E. De Man, G.D Khoe, and H. de Waardt, "Coherent equalization and POLMUX-RZ-DQPSK for robust 100-GE transmission," J. Lightwave Technol. 26(1), 64-72 (2008).

5. P. J. Winzer, and R. J. Essiambre, "Advanced modulation formats for high-capacity optical transport networks," J. Lightwave Technol. 24(12), 4711-4728 (2006)

6. M. S. Alfiad F. Machi, M. Kuschnerov, T. Wuth, D. van den Borne, N. Hanik, and H. de Waardt, "Feasibility study for $111 \mathrm{~Gb} / \mathrm{s}$ Polmux quadrature duobinary with a SE of $4.2 \mathrm{~b} / \mathrm{s} / \mathrm{Hz}$," OptoeElectronics and Communications Conference (OECC), 448-449, July (2010).

7. X. Zhou, J. Yu, M. F. Huang, Y. Shao, T. Wang, P. Magill, M. Cvijetic, L. Nelson, M. Birk, G. Zhang, S. Ten, H. B. Matthew, and S. K. Mishra, "Transmission of 32-Tb/s capacity over $580 \mathrm{~km}$ Using RZ-shaped PDM-8QAM modulation format and cascaded Mmltimodulus blind equalization algorithm," J. Lightwave Technol. 28(4), 456465 (2010).

8. A. Sano, E. Yamada, H. Masuda, E. Yamazaki, T. Kobayashi, E. Yoshida, Y. Miyamoto, R. Kudo, K.Ishihara, and Y. Takatori, "No-guard-interval coherent optical OFDM for 100-Gb/s long-haul WDM transmission," J. Lightwave Technol. 27(16), 3705-3713 (2009).

9. Y. Koizumi, K. Toyoda, M. Yoshida, and M. Nakazawa, "1024 QAM (60 Gbit/s) single-carrier coherent optical transmission over $150 \mathrm{~km}$," Opt. Express 20, 12508-12514 (2012).

10. A. Lender, "The duobinary technique for high speed data transmission," IEEE Trans. Commun. Electron. 82, 214-218 (1963).

11. M. E. Said, J. Sitch, and M. I. Elmasry, "An electrically pre-equalized 10-Gb/s duobinary transmission system," J. Lightwave Technol. 23, 388-400 (2005).

12. S. K. Ibrahim, S. Bhandare, and R. Noe, "Performance of $20 \mathrm{~Gb} / \mathrm{s}$ quaternary intensity modulation based on binary or duobinary modulation in two quadratures with unequal amplitudes," IEEE J. Sel. Top. Quantum Electron., 12(4), 596-602 (2006).

13. K. Kikuchi, Y. Ishikawa, and K. KATOH, "Coherent demodulation of optical quadrature duobinary signal with spectral efficien y of $4 \mathrm{bit} / \mathrm{s} / \mathrm{Hz}$ per polarization," 33rd European Conference and Ehxibition on Optical Communication (ECOC), P93.4, September (2007).

14. J. H. Chang, K. Y. Cho, H. Y. Hoi, Y. Takushima, and Y. C. Chung, "Filtering tolerance of 108-Gb/s polMux quadrature duobinary signal on 25-GHz grid," Optical Fiber Communication Conference and Exposition (OFC/NFOEC), OMR4, March (2011).

15. S. Zhang, F. Yaman, X. Lei, S. Yin, and W. Ting, "Generation of optical quadrature duobinary format using optical delay interferometer," 37th European Conference and Ehxibition on Optical Communication (ECOC), We.7.A.4, September (2011).

16. I. Lyubomirsky, "Quadrature duobinary for high-spectraleEfficien y 100G Transmission," J. Lightwave Technol. 28(1), 91-96 (2010).

17. F. Machi, M. S. Alfiad M. Kuschnerov, T. Wuth, D. van den Borne, N. Hanik, and H. de Waardt, "111-Gb/s polMux-quadrature duobinary for robust and bandwidth efficien Ttransmission," IEEE Photonics Technol. Lett. 22(11), 751-753 (2010)

18. R. Asif, C. Y. Lin, M. Holtmannspoetter, and B. Schmauss, "Optimized digital backward propagation for phase modulated signals in mixed-optical fibe transmission link," Opt. Express 18, 22796-22807 (2010).

19. R. Asif, C. Y. Lin, M. Holtmannspoetter, and B. Schmauss, "Multi-span digital non-linear compensation for dualpolarization quadrature phase shift keying long-haul communication systems," Opt. Commun. 285(7), 1814-1818 (2012).

20. R. Asif, C. Y. Lin, and B. Schmauss, "Impact of channel baud-rate on logarithmic digital backward propagation in DP-QPSK system with un-compensated transmission links," Opt. Commun. 284(24), 5673-5677 (2011).

21. C. Y. Lin, M. Holtmannspoetter, R. Asif, and B. Schmauss, "Compensation of transmission impairments by digital backward propagation for different link designs," 36th European Conference and Ehxibition on Optical Communication (ECOC), P3.16, September (2010).

22. L. Du, and A. Lowery, "Improved single channel back-propagation for intra-channel fibe non-linearity compensation in long-haul optical communication systems," Opt. Express 18, 17075-17088 (2010).

23. D. Rafique J. Zhao, and A. Ellis, "Digital back-propagation for spectrally efficien WDM 112 Gbit/s PM m-ary QAM transmission,” Opt. Express 19, 5219-5224 (2011).

24. C. Y. Lin, R. Asif, M. Holtmannspoetter, and B. Schmauss, "Step-size selection for split-step based nonlinear compensation with coherent detection in 112-Gb/s 16-QAM transmission," Chin. Opt. Lett 10, 020605 (2012).

25. R. Asif, C. Y. Lin, M. Holtmannspoetter, and B. Schmauss, "Low-complexity logarithmic step-size-based filtere digital backward propagation algorithm for compensating fibe transmission impairments," Proc. SPIE 8284 82840R (2012).

26. R. Asif, M. Usman, C. Y. Lin, and B. Schmauss, "Application of a digital non-linear compensation algorithm for evaluating the performance of root-raised-cosine pulses in $112 \mathrm{Gbit} / \mathrm{s}$ DP-QPSK transmission," J. Opt. 14(9), September (2012).

27. R. Asif, C.Y. Lin, and B. Schmauss, "Logarithmic versus modifie digital backward propagation algorithm in 224Gbit/s DP-16QAM transmission over dispersion uncompensated fibe links," Opt. Eng. 51(4), 045007 (2012). 
28. E. Mateo, M. Huang, F. Yaman, T. Wang, Y. Aono, and T. Tajima, "Nonlinearity compensation using very-low complexity backward propagation in dispersion managed links," Optical Fiber Communication Conference and Exposition (OFC/NFOEC), OTh3C.4, March (2011).

29. D. Rafique M. Mussolin, M. Forzati, J. Martensson, M. Chugtai, and A. Ellis, "Compensation of intra-channel nonlinear fibr impairments using simplifie digital back-propagation algorithm," Opt. Express 19, 9453-9460, April (2011).

30. L. Li, Z. Tao, L. Dou, W. Yan, S. Oda, T. Tanimura, T. Hoshida, and J. Rasmussen, "Implementation efficien non-linear equalizer based on correlated digital back-propagation," Conference on Optical Fiber communication/National Fiber Optic Engineers Conference (OFC/NFOEC) 2011, paper OWW3, Los Angeles USA, March (2011).

31. S. Zhang, F. Yaman, X. Lei, S. Yin, and M. Cvijetic, "Pulse shaping on quadrature duobinary format," IEEE Photonics Society Summer Topical Meeting Series, pp.149-150, 18-20th, July (2011).

\section{Introduction}

The requirements for bandwidth and capacity in metro and long-haul optical communication systems due to the demands of high-speed data services, internet video services, data centers and higher bandwidth residential broadband connections, are exponentially increasing. Recent advancements in high data-rate optical transmission systems have triggered an intensive investigation in digital signal processing (DSP) algorithms [1-3] as well as in spectrally efficien advanced modulation formats $[4,5]$. Coherent optical quadrature phase shift keying (CO-QPSK) is considered as the strongest candidate for implementing $100 \mathrm{GbE}$ networks due to its relatively low OSNR requirements and can have spectral efficien y (SE) of $2.2 \mathrm{~b} / \mathrm{s} / \mathrm{Hz}$ in case of DP-RZQPSK [6]. In order to meet the ever increasing demand of capacity requirements, increase in $\mathrm{SE}$ of the transmission system can be a possible solution. Most recently advanced modulation formats, M-quadrature amplitude modulation (QAM) $[\mathrm{M}=4,16,32,64,256$ and 1024] and orthogonal frequency-division multiplexing (OFDM) have been investigated in order to increase the SE [5,7-9]. However, this high SE is obtained at the expense of complicated transmitter structures, i.e. OFDM, and dense constellation diagrams, i.e. M-QAM (where M=16,32,64, 128 etc), which collectively make them less robust against fibe transmission impairments i.e. chromatic dispersion (CD) and non-linearities (NL).

Correlative coding (CC) or partial response signalling (PRS) techniques were firs introduced [10], in order to increase the spectral efficien y of the transmission systems. It was determined that by considering the correlation or dependencies between the amplitudes of transmitted pulses, we could achieve the symbol rate of $2 \mathrm{~N}$ symbols/s in a bandwidth of $\mathrm{N} \mathrm{Hz} \mathrm{[10].}$ This signalling method differs from the conventional pulse amplitude modulation (PAM) system in a sense that a controlled amount of inter-symbol interference (ISI) is introduced to attain certain spectral shapes which allows to transmit $2 \mathrm{~b} / \mathrm{s} / \mathrm{Hz}$ achieving full theoretical capability. This correlation between successive bits in a binary signal leads the signal spectrum to be more concentrated around the optical carrier [11]. Correlative coding technique is realized by implementing quadrature duobinary (QDB) modulation in recent times $[12,13]$ to attain high SE without increasing the complexity of the transmitter excessively $[14,15]$. The dual-polarization quadrature duobinary (DP-QDB) coding can have the SE of $4 \mathrm{~b} / \mathrm{s} / \mathrm{Hz}$ at $100 \mathrm{Gbit} / \mathrm{s}$ data rate and due to its relatively simple transmitter structure it becomes more attractive than DP-QAM [16].

The transmitter architecture of DP-QDB is relatively similar to DP-RZ-QPSK modulation, as shown in Fig. 1. The four electrical binary data streams driving the modulators are passed through duobinary pre-coders and then passed through a low pass filte (LPF) having the bandwidth of (symbol rate)/4. This LPF functions as delay and add components through the ISI, they induce between adjacent bits [17], as depicted in Fig. 1(a). For example, if the (input) data sequence is $x(n T)=(0,0,1,0,1,0,0,1,1,0)$, we would instead transmit the (output) data sequence $y(n T)=(0,0,1,0,1,0,0,1,1,0)+(*, 0,0,1,0,1,0,0,1,1)=(0,0,1,1,1,1$, $0,1,2,1)$. Here the sign * denotes the initial value $(z(n T))$ of the input sequence, which is

\#177330 - \$15.00 USD Received 1 Oct 2012; revised 15 Nov 2012; accepted 16 Nov 2012; published 8 Jan 2013

(C) 2013 OSA

14 January 2013 / Vol. 21, No. 1 / OPTICS EXPRESS 783 


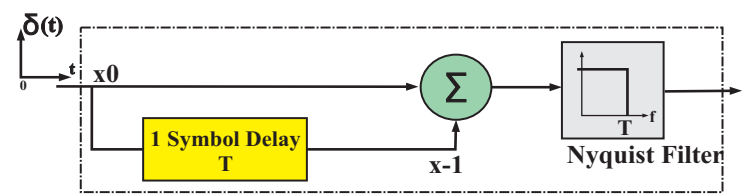

(a)

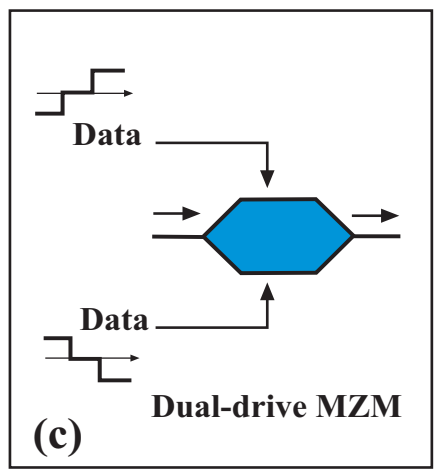

$\operatorname{Im}(\mathbf{E})$

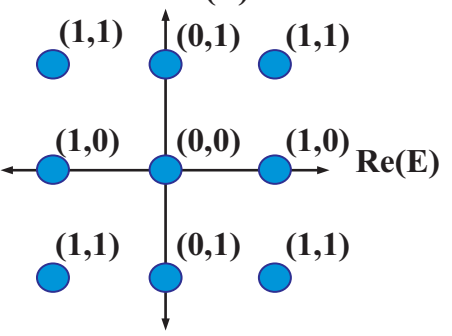

(e)
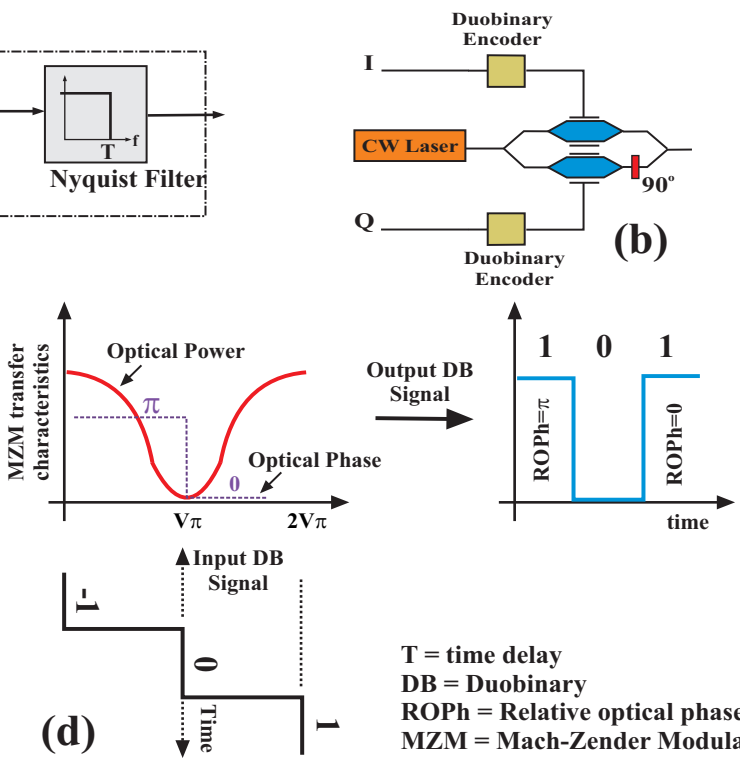

$T=$ time delay

DB = Duobinary

$\mathbf{R O P h}=$ Relative optical phase

MZM = Mach-Zender Modulator
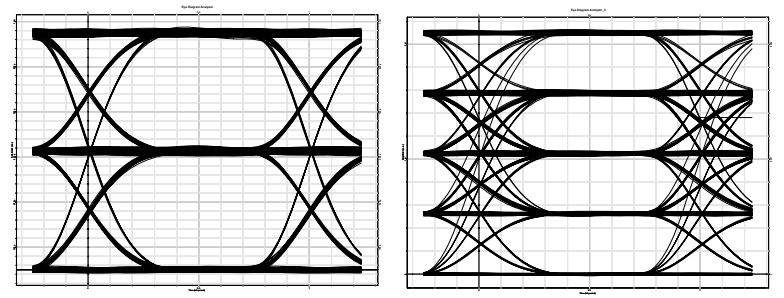

(f)

Fig. 1. (a) Equivalent model of filte stage for duobinary encoding, (b) Optical duobinary modulator, (c) Dual-derive MZM, (d) MZM bias and derive conditions for optical DB signal, (e) Quadrature duobinary (QDB) constellation diagram and (f) Eye-diagrams for DB and QDB respectively.

assumed to be zero. Note that while the input sequence is binary and consists of $0 \mathrm{~s}$ and $1 \mathrm{~s}$, the output sequence is a ternary sequence consisting of $0 \mathrm{~s}, 1 \mathrm{~s}$, and $2 \mathrm{~s}$. Mathematically, DB results in $y(n T)=x(n T)+x(n T-T)$, where $T$ is the bit period and $n$ in the number of bit sequences.

The DB signal can be generated by applying a baseband, three-level electrical DB signal to a dual-drive MZM as shown in Fig. 1(d) that is biased at maximum extinction ratio, as shown in Fig. 1(c). Conceptually, the carrier is a continuous wave signal, a sinusoid denoted by a. $\cos (w t)$. The three levels of the ternary signal correspond to a. $\cos (w t), 0$ and $-\mathrm{a} \cdot \cos (w t)$, which is denoted by $-1,0$, and +1 . These duobinary signals are then applied to the I-Q modulator, thus producing QDB signal (Fig. 1(b)). The 9-point constellation and eye-diagram of DB signal is shown in Fig. 1(e). The eye-diagram of QDB is also depicted in Fig. 1(f). The independently generated $x$ and $y$ polarized QDB signals are then combined by a polarization beam combiner to have DP-QDB.

Despite of all efforts to get the SE transmission with advanced modulation formats, CD and $\mathrm{NL}$ are the main impediments in improved system performance. To solve the issue, transmitter and receiver side digital signal processing (DSP) algorithms, termed as Digital Backward 
Propagation (DBP), to compensate chromatic dispersion and intra-channel non-linearities have been investigated in [18-26]. But the complexity of DBP algorithm is extremely high due to ultra-wide bandwidth requirements that it becomes an intrinsic impediment in the real-time implementation of DBP algorithm [27,28]. In order to reduce the complexity of the algorithm, the correlated DBP (CBP) algorithm has been investigated for QPSK transmission systems [29,30] and $80 \%$ reduction in the computational efficien y has been reported.

In this paper, we have numerically evaluated and compared the non-linear tolerance of correlative coding in the form of DP-QDB with DP-16QAM over 112Gbit/s transmission over $1640 \mathrm{~km}$ of large effective area pure-silica-core-fibe (LA-PSCF) link. The performance is evaluated both for single channel as well as 10-channel DWDM transmission with a channel spacing of $25 \mathrm{GHz}$. Furthermore, the comparison of transmission performance of DP-QDB and DP-RZQPSK modulation format has also been made at a channel spacing of $50 \mathrm{GHz}$ attaining a SE of $\mathrm{2b} / \mathrm{s} / \mathrm{Hz}$. The complexity of the DBP algorithm in-terms of step-size $(h)$ is also scrutinized for spectrally efficien modulation formats.

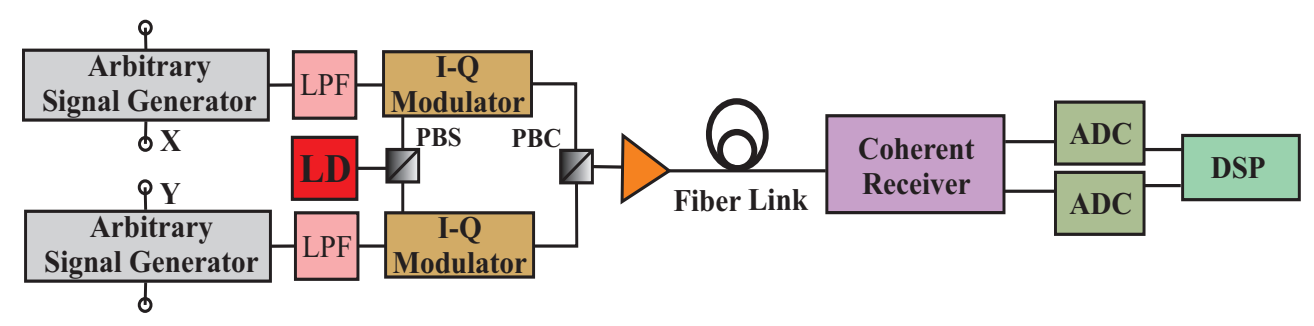

Fig. 2. Simulation setup for $112 \mathrm{Gbit} / \mathrm{s}$ DP-QDB system, whereas; LD=laser diode, $\mathrm{ADC}=$ analog to digital converter, $\mathrm{DSP}=$ digital signal processing module, $\mathrm{LPF}=$ low pass filte, $\mathrm{PBS}=$ polarization beam splitter, $\mathrm{PBC}=$ polarization beam combiner.

\section{Simulation setup}

Figure 2, illustrates the general numerical model to investigate the two modulation formats, i.e. DP-QDB and DP-16QAM, at a bit-rate of $112 \mathrm{Gbit} / \mathrm{s}$ for N-channels. The data streams consist of $2^{16}-1$ pseudo-random binary sequence (PRBS). The DP-16QAM transmitter is operated with four electrical binary streams with data rates of $14 \mathrm{Gbit} / \mathrm{s}$ for each polarization, whereas DP-QDB transmitter is modelled with four duobinary encoded electrical streams with data rates of $28 \mathrm{Gbit} / \mathrm{s}$ and RZ pulse shaping is also performed on DP-QDB [31]. In order to simulate DPRZ-QPSK system, RZ pulses with 50\% duty cycle are used and MZM is driven with a clock of $28 \mathrm{GHz}$. Single channel $(\mathrm{N}=1)$ and multi-channel $(\mathrm{N}=10)$ DWDM transmission performances are compared in this paper. The fibe link consists of Large $\mathrm{A}_{\text {eff }}$ Pure-Silica core fibe (LAPSCF) type fibe with 20 spans of $82 \mathrm{~km}$ each and has the physical parameters of: attenuation $\alpha=0.16 \mathrm{~dB} / \mathrm{km}$, dispersion $D=21 \mathrm{ps} /(\mathrm{nm}-\mathrm{km})$ and non-linear coefficien $\gamma=0.6\left(\mathrm{~km}^{-1} \cdot \mathrm{W}^{-1}\right)$. No in-line optical dispersion compensator is employed in the link. Erbium-doped fibe amplifier (EDFAs) are modelled with $13.12 \mathrm{~dB}$ of gain and $4 \mathrm{~dB}$ of noise figure A phase-diversity homodyne coherent receiver is used to detect the DP-16QAM and DP-QDB [17]. To simplify our numerical analysis, we neglect the effect of polarization mode dispersion (PMD) and laser line width. In order to investigate the transmission performance of DWDM DP-QDB and DP$16 \mathrm{QAM}$ at $4 \mathrm{~b} / \mathrm{s} / \mathrm{Hz} \mathrm{SE}, 10$ transmitters are multiplexed with $25 \mathrm{GHz}$ channel spacing. While for the investigations of $2 \mathrm{~b} / \mathrm{s} / \mathrm{Hz}$ SE for DP-RZ-QPSK and DP-QDB transmission, 10 transmitters are multiplexed with $50 \mathrm{GHz}$ channel spacing The system performances are evaluated by monitoring the bit-error-ratio (BER) and the forward error correction (FEC) limit corresponds

\#177330 - \$15.00 USD Received 1 Oct 2012; revised 15 Nov 2012; accepted 16 Nov 2012; published 8 Jan 2013

(C) 2013 OSA

14 January 2013 / Vol. 21, No. 1 / OPTICS EXPRESS 785 
to BER of $3.8 \times 10^{-3}$. The DBP processing module is used after the coherent detection and is based on the symmetric split-step Fourier method.

\section{Results and discussions}

\subsection{Tolerance to non-linear transmission impairments}

Figure 3(a), depicts the transmission performance of DP-16QAM 112Gbit/s transmission over $1640 \mathrm{~km}$ of fibe. The system performance is compared by digitally compensating the fibe transmission impairments as; (a) chromatic dispersion compensation (CD), i.e. linear equalization (LE) and (b) digital backward propagation (DBP). The DBP algorithm is employed here on the basis of one-step per fibe span (DBP-1S). The single channel transmission shows improvement in system performance in terms of input launch power by a factor of $\approx 2.8 \mathrm{~dB}$ and $\approx 2.1 \mathrm{~dB}$ in DWDM transmission at BER of $3.8 \times 10^{-3}$. This improvement depicts efficien compensation of CD and NL by DBP. Whereas; the reduced improvement in case of DWDM is due to the strong inter-channel non-linear effects.
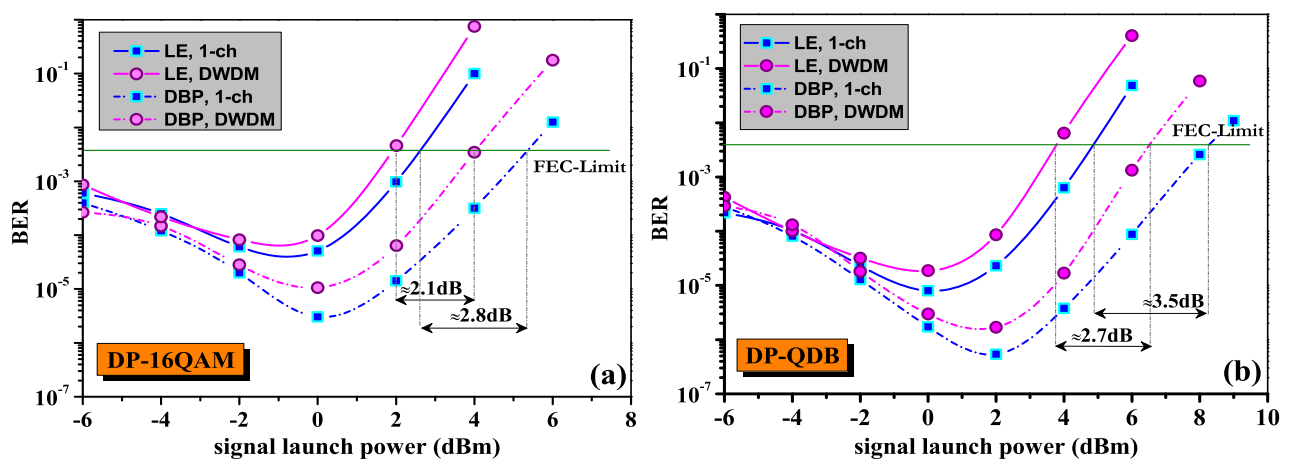

Fig. 3. BER performance of 112Gbit/s: (a) DP-16QAM and (b) DP-QDB (right) for single channel (1-ch) and multi-channel (DWDM) with $25 \mathrm{GHz}$ channel spacing using; (i) linear equalization (LE) and (ii) digital backward propagation (DBP).

The tolerance of non-linear transmission in DP-QDB system is analysed in Fig. 3(b). We have investigated LE and DBP performance in this case for the same link design as our previous investigation. We have seen that the system performance is improved by a factor of $\approx 3.5 \mathrm{~dB}$ in single channel and $\approx 2.7 \mathrm{~dB}$ in DWDM transmission. Also that the overall non-linear threshold point (NLT) is improved in DP-QDB by 2dB as compared to DP-16QAM. This depicts that DP-QDB is more tolerant to non-linear effects, shows enhanced system performance with DBP and can transmit over longer distances as compared to DP-16QAM system, while both have same spectral efficien y (SE) of $4 \mathrm{~b} / \mathrm{s} / \mathrm{Hz}$. On the other hand another added advantage of DPQDB modulation is its feasibility of realizing high SE without increasing the complexity of the transmitter excessively.

Furthermore, we have numerically evaluated and compared the transmission performances at different spectral efficiencies For this investigation a 10 channel DP-QDB transmission system is implemented with $50 \mathrm{GHz}$ channel spacing giving a SE of $2 \mathrm{~b} / \mathrm{s} / \mathrm{Hz}$. The performance is compared with DP-RZ-QPSK transmission. The other physical parameters of the transmission set-up are kept constant as previous investigation. The results are depicted in Fig. 4 after implementing the DBP algorithm. It is clear from the results that DP-RZ-QPSK has more nonlinear tolerance as compared to DP-QDB at a SE of $2 \mathrm{~b} / \mathrm{s} / \mathrm{Hz}$ and the system performance has improved by $\approx 3 \mathrm{~dB}$ at $\mathrm{BER}$ of $1 \times 10^{-3}$. It means that for $50 \mathrm{GHz}$ channel spacing, the DP-RZ- 
QPSK modulation has more tolerance for non-linear transmission impairments, have the simple transmitter/receiver architectures and less complex signal processing algorithms to detect the received signals, as compared to DP-QDB. But at higher SE, DP-QDB can be used to reduce the channel spacing to $25 \mathrm{GHz}$ in order to obtain an SE of 4-b/s/Hz.

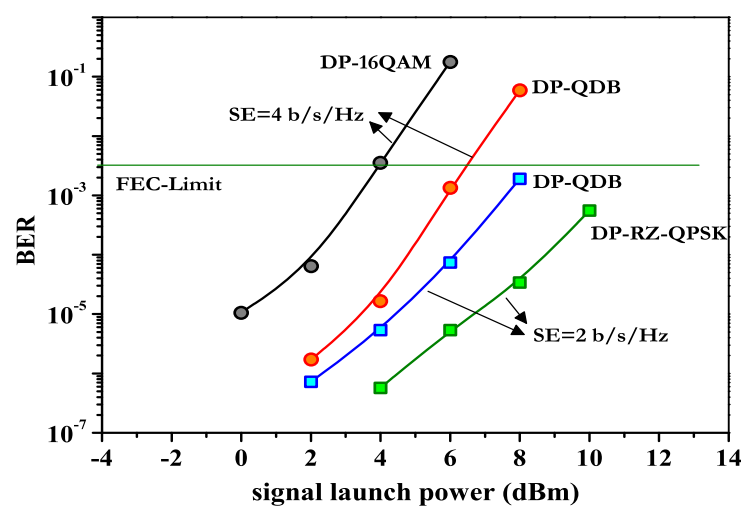

Fig. 4. Non-linear transmission performance of 10-channel DP-QDB vs. DP-16QAM (with $25 \mathrm{GHz}$ channel spacing) and DP-QDB vs. DP-RZ-QPSK (with $50 \mathrm{GHz}$ channel spacing).

\subsection{Complexity of DBP algorithm for spectrally efficient modulation formats}

In order to exploit the complexity of the DBP algorithm for both the modulation formats, we have investigated the performance w.r.t different DBP step number per $1640 \mathrm{~km}$ of fibe link. The $112 \mathrm{Gbit} / \mathrm{s}$ system is investigated at $4 \mathrm{dBm}$ signal launch power and the results are depicted in Fig. 5. The results illustrate a significan decrease in the complexity of the DBP algorithm for single channel DP-QDB encoded transmission. The comparative system performance w.r.t DP-16QAM transmission can be obtained in just 8 DBP steps for $1640 \mathrm{~km}$ of fibe link $(60 \%$ reduction in algorithm complexity). This computational effort corresponds to $0.4 \mathrm{DBP}$ steps per fibe span and $205 \mathrm{~km}$ of step-size. Furthermore; for DWDM transmission at the FEC limit (BER of 3.8 $\times 10^{-3}$ ), by using DP-QDB encoded transmission, the complexity of the DBP algorithm can be reduced to 0.7 steps per fibe span (30\% reduction) w.r.t DP-16QAM transmission. The overall results depict that; (a) DP-QDB transmission has more non-linear tolerance as compared to DP-16QAM and (b) the low-complexity of the DBP algorithm can be achieved by utilizing DP-QDB transmission both in single and multi-channel transmission. Even at the NLT point, DP-QDB is performing efficientl , as shown in Fig. 3(b), compared to DP-16QAM, so we will get the reduced complexity likewise.

Finally, we have focused on the numerical investigation of maximum achievable transmission distance for a BER of $1 \times 10^{-3}$ employing DBP in the transmission for both the modulation formats. Using the same parameters of transmission i.e. $112 \mathrm{Gbit} / \mathrm{s}, 1640 \mathrm{~km}$ of fibe and by using DBP-1S, the results are as shown in Fig. 6. By analysing the results we have noticed that DP-QDB encoded transmission has the maximum transmission reach of $5280 \mathrm{~km}$ and $4900 \mathrm{~km}$ for single and multi-channel transmission respectively. This actually out performs the DP-16QAM transmission which has the maximum transmission reach of approximately $4500 \mathrm{~km}$ and $4200 \mathrm{~km}$. We have found from our numerical investigations that we can achieve $15-18 \%$ more transmission distance in case of DP-QDB encoded transmission. This shows once again the superior performance of DP-QDB over DP-16QAM. 


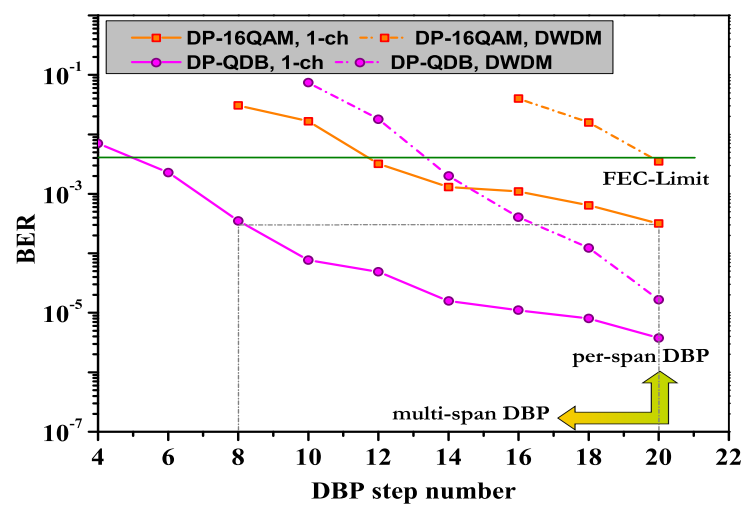

Fig. 5. BER as a function of number of DBP calculation steps for complete fibe link.

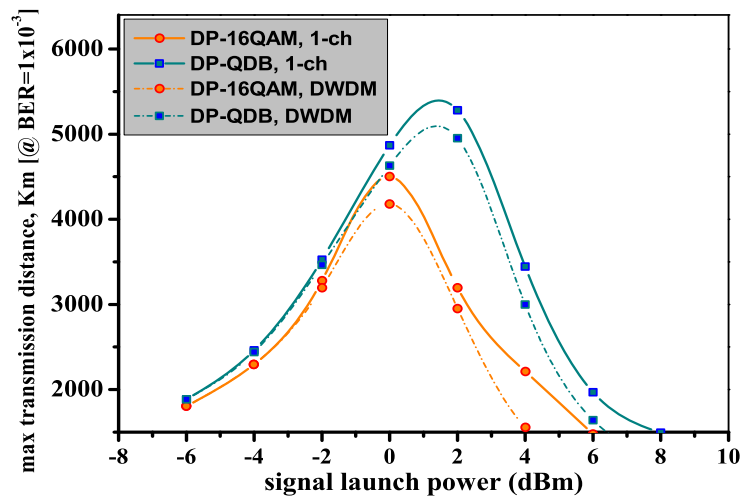

Fig. 6. Optical transmission reach at BER of $1 \times 10^{-3}$ for DP-QDB and DP-16QAM transmission.

\section{Conclusion}

In this paper, we have numerically evaluated the transmission performance of $112 \mathrm{Gbit} / \mathrm{s}$ N-channel Correlative encoded (DP-QDB) and DP-16QAM systems employing DBP over $1640 \mathrm{~km}$ of fibe with no in-line optical dispersion compensation. The DP-QDB encoded transmission is investigated for single and multi-channel transmission, complexity of DBP algorithm and maximum optical transmission reach. We have also numerically evaluated and compared the transmission performances at different spectral efficiencies i,e. $2 \mathrm{~b} / \mathrm{s} / \mathrm{Hz}$ and $4 \mathrm{~b} / \mathrm{s} / \mathrm{Hz}$. While comparing to DP-16QAM with the same data rate and SE, DP-QDB has a transmitter structure similar to DP-QPSK and a better tolerance against non-linearities. In case of DP-QDB transmission; (a) NLT is improved by a factor of $2 \mathrm{~dB}$, (b) the complexity of DBP algorithm is reduced by $60 \%$ [0.4DBP steps per fibe span, step-size of $205 \mathrm{~km}]$ and (c) maximum transmission reach is improved by $15-18 \%$. The inclusive results depict promising performance of DP-QDB for reducing the complexity of DBP algorithm.

\section{Acknowledgement}

The authors gratefully acknowledge funding of the Erlangen Graduate School in Advanced Optical Technologies (SAOT) by the German National Science Foundation (DFG) in the framework of the excellence initiative.

\#177330 - \$15.00 USD Received 1 Oct 2012; revised 15 Nov 2012; accepted 16 Nov 2012; published 8 Jan 2013

(C) 2013 OSA

14 January 2013 / Vol. 21, No. 1 / OPTICS EXPRESS 788 